\title{
Neurodegeneration and Motor Deficits in the Absence of Astrogliosis upon Transgenic Mutant TDP-43 Expression in Mature Mice
}

\author{
Gabriella Chan, Annika van Hummel, Julia van der Hoven, Lars M. Ittner, and Yazi D. Ke
}

From the Dementia Research Centre and Department of Biomedical Sciences, Faculty of Medicine, Health and Human Sciences, Macquarie University, Sydney, NSW, Australia

\author{
Accepted for publication \\ April 16, 2020. \\ Address correspondence to Yazi \\ D. Ke, Ph.D., Dementia \\ Research Centre, Faculty of \\ Medicine, Health and Human \\ Sciences, Macquarie University, \\ 13A Research Park Dr., Sydney, \\ NSW 2109, Australia. E-mail: \\ yazi.ke@mq.edu.au.
}

\begin{abstract}
Amyotrophic lateral sclerosis is a rapidly progressing and fatal disease characterized by muscular atrophy due to loss of upper and lower motor neurons. Pathogenic mutations in the TARDBP gene encoding TAR DNA binding protein-43 (TDP-43) have been identified in familial amyotrophic lateral sclerosis. We have previously reported transgenic mice with neuronal expression of human TDP-43 carrying the pathogenic A315T mutation (iTDP-43 ${ }^{\mathrm{A} 315 \mathrm{~T}}$ mice) using a tetracycline-controlled inducible promotor system. Constitutive expression of transgenic TDP-43 ${ }^{\mathrm{A} 315 \mathrm{~T}}$ in the absence of doxycycline resulted in pronounced early-onset and progressive neurodegeneration, and motor and memory deficits. Here, delayed transgene expression of TDP $-43^{\mathrm{A} 315 \mathrm{~T}}$ by oral doxycycline treatment of $\mathrm{iTDP}-43^{\mathrm{A} 315 \mathrm{~T}}$ mice from birth till weaning was analyzed. After doxycycline withdrawal, transgenic TDP-43 ${ }^{\mathrm{A} 315 \mathrm{~T}}$ expression gradually increased and resulted in cytoplasmic TDP-43, widespread ubiquitination, and cortical and hippocampal atrophy. In addition, these mice developed motor and gait deficits with underlying muscle atrophy, similar to that observed in the constitutive iTDP-43 ${ }^{\mathrm{A} 315 \mathrm{~T}}$ mice. Surprisingly, in contrast to the constitutive iTDP-43 ${ }^{\mathrm{A} 315 \mathrm{~T}}$ mice, these mice did not develop astrogliosis. In summary, delayed activation coupled with gradual increase in TDP-43 ${ }^{\text {A315T }}$ expression in the central nervous system of mature mice resulted in progressive functional deficits with neuron and muscle loss, but in the absence of a glial response. This suggests that astrocytosis does not contribute to functional deficits and neuronal loss upon TDP-43 ${ }^{\mathrm{A} 315 \mathrm{~T}}$ expression in mature mice. (Am J Pathol 2020, 190: 1713-1722; https://doi.org/10.1016/j.ajpath.2020.04.009)
\end{abstract}

Amyotrophic lateral sclerosis (ALS), also known as motor neurone disease or Lou Gehrig's disease, is a rapidly progressive and fatal neurodegenerative condition affecting specific nerve cells of the spinal cord, brain stem, and brain. Although progressive muscle weakness and nerve cell degeneration are the main symptoms of ALS, the disease shares symptoms, neuropathology, and genetics with frontotemporal lobar degeneration (FTLD) as part of a disease continuum. ${ }^{1}$ The neuropathologic hallmark shared by the majority of ALS and approximately half of FTLD cases is ubiquitinated protein deposits in neurons containing TAR DNA-binding protein 43 (TDP-43). ${ }^{2}$ Furthermore, TDP-43 pathology has been described in a large proportion of Alzheimer disease cases, further implicating its role in neurodegeneration. ${ }^{3}$

TDP-43 localizes predominantly to the nucleus of cells under physiological conditions, where it is involved in gene transcription, pre-mRNA splicing, and mRNA stability and transport. ${ }^{4}$ Nuclear localization and export sequences within the amino-terminal of TDP-43 mediate its nuclearcytoplasmic localization. ${ }^{5}$ In disease, TDP-43 becomes increasingly phosphorylated and ubiquitinated, and accumulates in the cytoplasm of neurons, forming inclusions. ${ }^{2}$ There is increasing evidence that soluble and cleaved

\footnotetext{
Supported by Motor Neuron Disease Australia grant GIA1824 (L.M.I.); the Australian National Health \& Medical Research Council (NHMRC) grants 1081916, 1132524, 1143978 and 1143848 (L.M.I. and Y.D.K.); the Australian Research Council grants DP170100781 and DP170100843 (L.M.I.); and Macquarie University (L.M.I. and Y.D.K.). Y.D.K. is a NHMRC Career Development R.D. Wright Biomedical Fellow grant 1123564). L.M.I. is a NHMRC Principal Research Fellow (grant 1136241).

Disclosures: None declared.
} 
species of TDP-43 significantly contribute to neuronal dysfunction and disease processes. ${ }^{6}$ Similarly, loss of physiological functions, that is, haploinsufficiency, in the nucleus may contribute to disease onset and progression.

Mutations in the TDP-43-encoding gene TARDBP have been identified in a small subset of familial ALS cases, further supporting an important role in disease. Furthermore, TDP-43 pathology is common in sporadic and familial ALS, as well as in FTLD with pathogenic mutations in several other genes such as C9ORF72, GRN, and VCP. ${ }^{8}$

The discovery that TDP-43 is a major component of ubiquitinated inclusions in ALS together with the identification of pathogenic and functional mutations in TARDBP have facilitated the generation of transgenic TDP-43 mouse models. ${ }^{9,10}$ Since the initial identification of TDP-43 mutations in ALS, a plethora of both mutant and nonmutant TDP-43 have been expressed in mice using different promoters to model aspects of ALS in vivo. ${ }^{8}$ The phenotypic, biochemical, and histologic recapitulation of the disease varies between different TDP-43 transgenic lines, with some neuropathologic features, functional deficits, neuronal loss, and reduced survival being reproduced. Given the prevalence of TDP-43 pathology in ALS, transgenic TDP-43 mouse models are now more widely used in studying disease mechanisms, as well as testing of novel therapeutic approaches. ${ }^{11}$ Detailed characterization of phenotype progression, and biochemical and neuropathologic features in individual TDP-43 transgenic lines is critical and vital in the quest to better understand disease mechanisms.

We have previously reported early-onset motor and behavioral deficits in young transgenic mice expressing human TDP-43 with the pathogenic familial A315T mutation in neurons (iTDP- $\left.43^{\mathrm{A} 315 \mathrm{~T}}\right) .{ }^{10}$ In addition, a selective vulnerability of upper motor neurons to pathologic TDP- $43^{\mathrm{A} 315 \mathrm{~T}}$ was further identified when aged iTDP-43 ${ }^{\mathrm{A} 315 \mathrm{~T}}$ mice were analyzed. ${ }^{12}$ Both previous studies are in iTDP- $43^{\mathrm{A} 315 \mathrm{~T}}$ mice with constitutive transgene expression. In this report, the delayed expression of this human TDP-43 $3^{\mathrm{A} 315 \mathrm{~T}}$ is further characterized by transgene suppression through oral doxycycline treatment from gestation to weaning. This delayed TDP-43 ${ }^{\mathrm{A} 315 \mathrm{~T}}$ expression resulted in recapitulation of several neuropathologic, biochemical, and phenotypic features similar to constitutive expressing iTDP-43 ${ }^{\mathrm{A} 315 \mathrm{~T}}$ mice, but this occurred in the absence of astrogliosis.

\section{Materials and Methods}

\section{Mice}

Transgenic mice with expression of neuronal transactivator [line mThy1.2-tTA(6)] and human A315T mutant TDP-43 under control of the tetracycline-responsive element promoter [line pTRE-TDP-43 ${ }^{\mathrm{A} 315 \mathrm{~T}}(13)$ ] have been described previously. ${ }^{10}$ To switch off the expression of transgene till weaning (3-week-old), breeders and their offspring were fed standard chow supplemented with doxycycline $(200 \mathrm{mg} /$ kg; Specialty Feeds SF11-059; Specialty Feeds, Glen Forrest, WA). ${ }^{13}$ All mice are henceforth termed diTDP$43^{\mathrm{A} 315 \mathrm{~T}}$. After weaning, mice were placed in cages with access to regular chow to allow transgene expression. All mice were maintained on a C57Bl/6 background and housed on a 12-hour light/dark cycle with access to standard chow (post-weaning) and water ad libitum. Mice of both sexes were used throughout this study. All experiments were approved by the Macquarie University animal care and ethics committee.

\section{Gait Analysis}

Gait of mice was analyzed using a DigiGait system (Mouse Specifics, Boston, MA). Briefly, individual mice were placed in an enclosed chamber on a transparent, motorized treadmill belt and allowed to acclimatize to the machine at a speed of $6 \mathrm{~cm} / \mathrm{second}$. Mice were then recorded for at least four complete strides at $15-$ to $17-\mathrm{cm} / \mathrm{second}$ belt speed, with the paws captured by a camera from underneath. Gait analysis was performed in 15-month-old mice $(n=13$ [single or nontransgenic (non-tg)], $n=12$ [iTDP-43]). A range of spatial and temporal gait parameters was calculated by DigiGait software (DigiGait Analyser v14.5, DigiGait Imager v14; Mouse Specifics, Framingham, MA) for each limb to detect differences in gait between transgenic and non-tg mice.

\section{Motor Testing}

All motor tests were performed as previously described ${ }^{10}$ with $n=14$ (single or non-tg) and $n=13$ (iTDP-43) 8to 11-month-old diTDP-43 ${ }^{\text {A315T }}$ mice. Rota-Rod: Motor performance of mice was determined using a rotarod (Ugo Basile, Varese, Italy) in acceleration mode (5 to $60 \mathrm{rpm}$ ) over 120 seconds. The longest time each mouse remained on the turning wheel out of five attempts per session was recorded. Mice were previously recorded daily over 10 days and five trials per day, with no differences observed between mice trained longer or shorter on the rotarod. As such, only the 3 days of testing were done for the rest of the study. Pole test: To test strength and coordination, mice were placed at the apex of a vertical pole $(47.5-\mathrm{cm}$ length of dowel, diameter $0.8 \mathrm{~cm}$ ) facing upward. The time taken to turn around, descend the pole, and reach the ground (with all four paws) was measured with a maximum time of 120 seconds. Mice underwent one to two training sessions prior to the test session, during which the best time was taken out of two trials. Mice who were unable to descend the pole (slipped or fell) were given the maximum time. Hanging wire test: Mice were allowed to hang upside down on an inverted rectangular wire mesh over a plexiglass box for a maximum of 3 minutes, and latency to falling off was recorded (longest time out of two attempts). ${ }^{12}$ Grip strength test: A grip strength meter (Chatillon; AMETEK, Berwyn, PA) was used to measure the force exerted by the forelimbs 
of the mouse. Mice were placed such that they had a double overhand grip on a thin metal wire attached to the meter, and were pulled away from the meter in a horizontal direction until they let go; a peak force $(\mathrm{N})$ was recorded at the moment when the mice let go. The highest force from five attempts was recorded.

\section{Protein Extraction and Western Blot}

Proteins were sequentially extracted based on solubility from 90 to $120 \mathrm{mg}$ of cortical tissue, and immunoblotting was performed on extracts as previously described. ${ }^{10}$ Proteins were sequentially extracted in $10 \mu \mathrm{L} / \mathrm{mg}$ radioimmunoprecipitation assay (RIPA) buffer [0.1\% SDS, 1\% NP40, 0.5\% sodium deoxycholate, $5 \mathrm{mmol} / \mathrm{L}$ EDTA, $150 \mathrm{mmol} / \mathrm{L} \mathrm{NaCl}, 50 \mathrm{mmol} /$ $\mathrm{L}$ Tris-HCl (pH 8.0), $30 \mathrm{mmol} / \mathrm{L} \mathrm{NaF}, 60 \mathrm{mmol} / \mathrm{L} \beta$-glycerophosphate, $20 \mathrm{mmol} / \mathrm{L}$ sodium pyrophosphate, $1 \mathrm{mmol} / \mathrm{L}$ sodium orthovanadate, and protease inhibitor (Roche, Basel, Switzerland)], sonicated at $20 \%$ amplitude, incubated at $4^{\circ} \mathrm{C}$ for 30 minutes, and centrifuged at $50,000 \times g, 4^{\circ} \mathrm{C}$ for 1 hour. The supernatant was collected as the RIPA fraction, and 2 additional RIPA washes were performed. The pellet was resuspended in urea buffer [ $7 \mathrm{~mol} / \mathrm{L}$ urea, $2 \mathrm{~mol} / \mathrm{L}$ thiourea, $4 \%$ CHAPS, $3 \mathrm{mmol} / \mathrm{L}$ Tris ( $\mathrm{pH} \mathrm{8.5)]} \mathrm{and} \mathrm{sonicated} \mathrm{as} \mathrm{the} \mathrm{urea}$ fraction. Protein concentration of extracts was determined using the Bradford assay (Bio-Rad Laboratories, Hercules, $\mathrm{CA}$ ), and 20 to $40 \mu \mathrm{g}$ of protein was resolved on $10 \%$ to $15 \%$ SDS-PAGE gels. After separation, proteins were semidry transferred onto nitrocellulose membranes (GE Healthcare, Chicago, IL). Membranes were blocked with bovine serum albumin (Invitrogen; Thermo Fisher Australia, Mulgrave, VIC, Australia), and probed with antibodies against human TDP-43 (60019-2-Ig; Proteintech, Rosemont, IL), mouse TDP-43 (a gift from Prof. Virginia Lee, University of Pennsylvania), phospho TDP-43 Ser409/410 (TIP-PTD-P02; Cosmo Bio USA, Tokyo, Japan), and GAPDH (MAB374; MilliporeSigma, Burlington, MA). Blots were then incubated with horseradish peroxidase (HRP)-conjugated secondary antibodies, visualized using Luminata Crescendo Western HRP Substrate (MilliporeSigma) and imaged with the ChemiDoc MP System (Bio-Rad Laboratories).

\section{Histology and Staining}

For immunohistochemistry, tissues were embedded in paraffin and then sectioned. Staining of sectioned tissue was done as previously described. ${ }^{14}$ Fifteen-month-old mice were used for all histologic analyses $[n=13$ (single or non$\operatorname{tg}$ ) and $n=12$ (iTDP-43)]. The following primary antibodies were used: human TDP-43 (60019-2-Ig; Proteintech), ubiquitin (MAB1510; MilliporeSigma), POU3F2 (ab94977; Abcam, Cambridge, UK), glial fibrillary acidic protein (GFAP) (G9269; Sigma-Aldrich, St. Louis, MO), Iba-1 (01919741; Wako Chemicals, Osaka, Japan), and laminin (L9393; Sigma-Aldich). For chromogenic detection, the following secondary antibodies and detection kits were used; mouse-biotinylated secondary antibodies (Life Technologies, Carlsbad, CA) together with avidin-biotin complex HRP detection kit (ABC-HRP; Vector Laboratories, Burlingame, CA). For immunofluorescence, Alexa Fluor 488 and 555 (Life Technologies) antibodies together with DAPI for nucleus were used.

\section{Image Analysis}

Stained sections were imaged using BX51 epifluorescence microscope with DP70 color camera and CellSens software version 2.2 (Olympus, Tokyo, Japan) or the AxioScan.Z1 slide scanner with ZEN 2.6 software blue edition (Carl Zeiss, Oberkochen, Germany). Images were then quantified using FIJI ImageJ software version 1.52p (NIH, Bethesda, MD; https://imagej.nih.gov/ij). ${ }^{15}$

\section{Statistical Analysis}

Statistical analysis was done using the Prism software package version 7.02 (GraphPad Software, San Diego, CA). The pole, balance beam, hanging wire, and grip strength tests, and DigiGait data were analyzed using $t$-test. RotaRod analysis was done using two-way analysis of variance. Single- and non-tg mice were combined as controls for histologic analysis and compared with transgenic mice using $t$-test or analysis of variance. All post hoc analysis was done using Sidak multiple comparisons. All values are expressed as means \pm SEM.

\section{Results}

Delayed TDP-43 ${ }^{\mathrm{A} 315 \mathrm{~T}}$ Expression Results in Cytoplasmic Neuronal Accumulation

To study the impact of human mutant TDP-43 expression after brain maturation, the authors used their previously developed A315T mutant TDP-43-expressing inducible iTDP- $43^{\mathrm{A} 315 \mathrm{~T}}$ mice $^{10}$ and suppressed transgene expression from gestation to weaning via oral doxycycline (henceforth termed diTDP-43) followed by neuropathologic and functional analysis up to 15 months of age (Figure 1A). Throughout the study, diTDP-43 mice were compared with littermate controls that included single transgenic (pTRE$\mathrm{TDP}^{\mathrm{A} 315 \mathrm{~T}}$ or mThy 1.2-tTA) or non-tg mice. Transgene suppression was confirmed at 1 month of age with a gradual increase in the number of TDP- $43^{\mathrm{A} 315 \mathrm{~T}}$ - positive cortical cells from 4 to 12 months of age observed (Figure 1B). At the cellular level, this gradual increase in transgene expression occurs in conjunction with a concomitant loss of endogenous murine TDP-43 (Figure 1B), similar to that observed in constitutive expression of the transgene in iTDP- $43^{\mathrm{A} 315 \mathrm{~T}} .{ }^{10}$ Using the pan-neuronal tetR expression system under a murine Thy 1.2 promoter, TDP- $43^{\mathrm{A} 315 \mathrm{~T}}$ expression was achieved throughout the fore-, mid-, and hindbrain, and spinal cord of iTDP- $43^{\mathrm{A} 315 \mathrm{~T}}$ mice after cessation of doxycycline treatment 


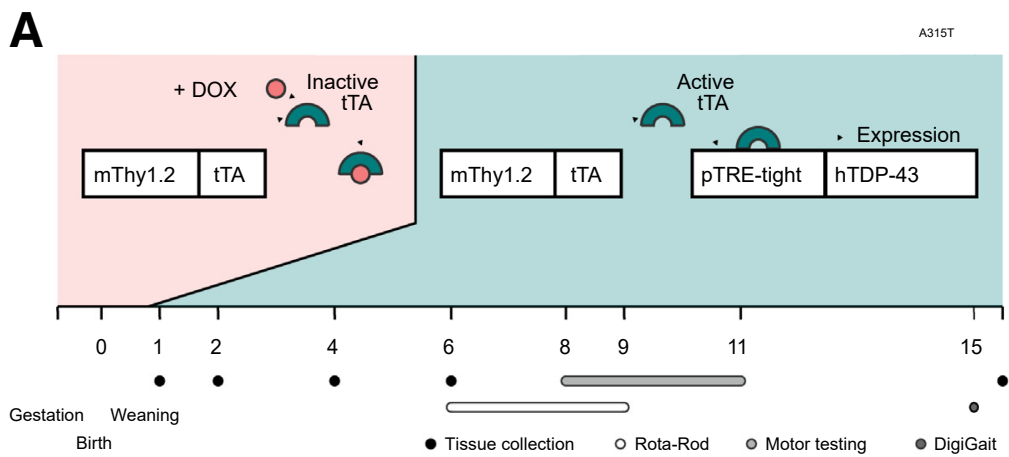

B
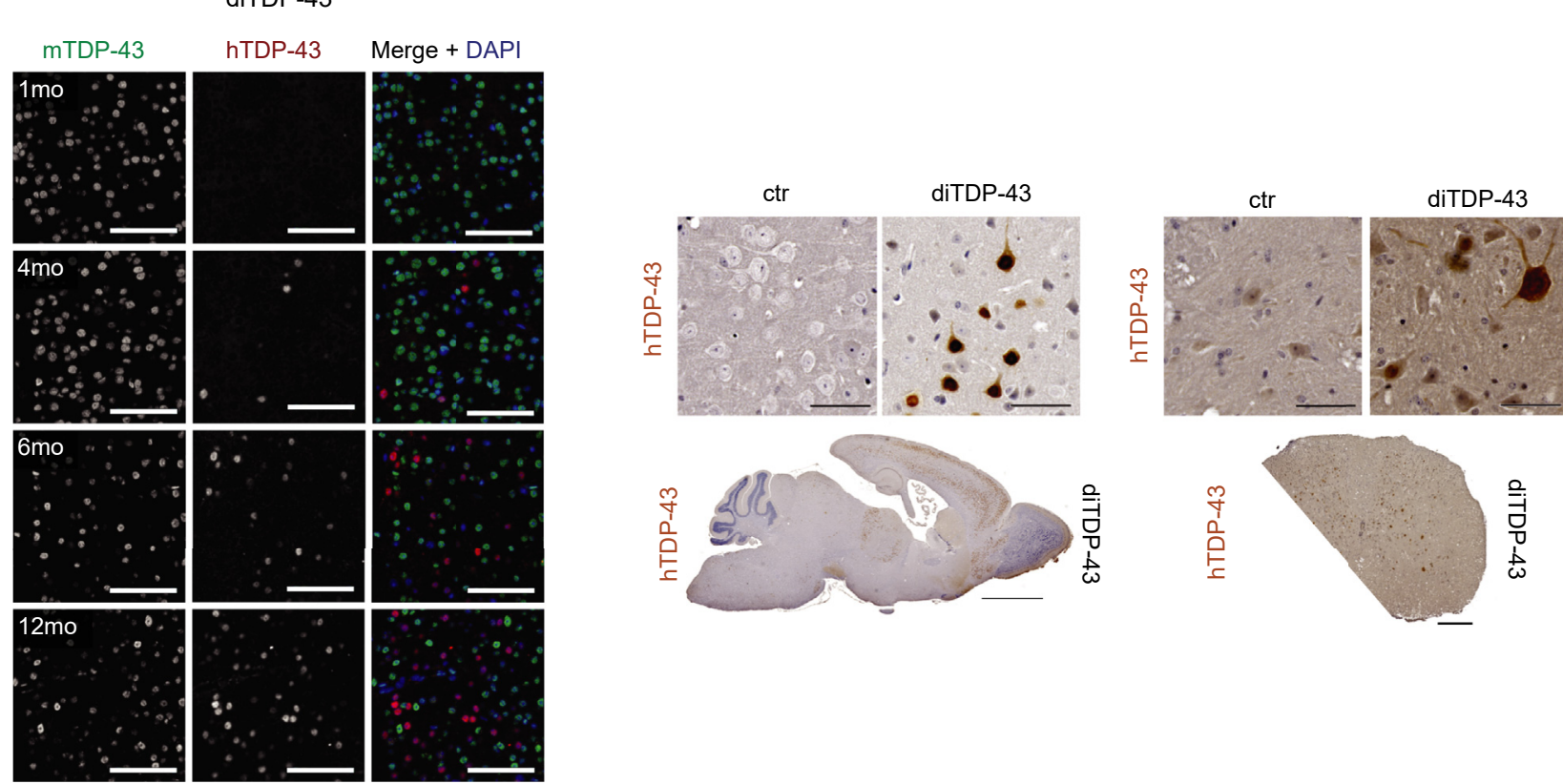

Figure 1 A: Doxycycline (DOX) treatment timeline. Transgene suppression was achieved from gestation to weaning via oral doxycycline in chow. Doxycycline was removed after weaning and neuronal hTDP-43 ${ }^{\mathrm{A} 15 \mathrm{~T}}$ transgene expression was turned on. Behavioral analysis was performed from 9 months (mo) of age, and tissues were subsequently collected for histology. B: Double immunofluorescence staining with antibodies to human TAR DNA binding protein 43 (hTDP-43; red) and murine TDP-43 (mTDP-43; green) confirms transgene suppression at 1 month of age with a gradual increase in hTDP-43 over time as depicted at 4, 6, and 12 months. Transgene hTDP-43 expression is virtually mutually exclusive to mTDP-43, consistent with a down-regulation of mTDP-43 in mutant hTDP-43-expressing cells. C: Immunohistochemical staining of human TDP-43 (hTDP-43; brown) of sagittal whole-brain sections shows strong transgene in the cortex, hippocampus, and midbrain of transgenic diTDP-43 mice (below). High magnification shows presence of cytoplasmic hTDP-43 (top) in diTDP-43 as compared with control (ctr). D: Immunohistochemical staining of human TDP-43 (hTDP-43, brown) of transverse sections of spinal cord shows transgenic hTDP-43 expression throughout all neuronal cell types. Scale bars: $50 \mu \mathrm{m}$ (B, and C and D, top row); $2 \mu \mathrm{m}$ (C, bottom panel); $200 \mu \mathrm{m}$ (D, bottom panel). tTA, tetracycline-controlled transactivator.

(Figure 1, C and D). Immunohistochemistry showed that transgenic human TDP-43 ${ }^{\mathrm{A} 315 \mathrm{~T}}$ expressed in cortical neurons localized to both nuclei and cytoplasm (Figure 1C). Similarly, spinal cord neurons showed cytoplasmic accumulation of human transgenic TDP-43 ${ }^{\mathrm{A} 315 \mathrm{~T}}$ in addition to neuronal expression (Figure 1D). No human TDP-43 expression was detected in brains and spinal cords of control mice. In short, following doxycycline suppression of transgenic TDP$43^{\mathrm{A} 315 \mathrm{~T}}$ expression during development, a gradual increase in expression resulted in cytoplasmic localization of mutant TDP-43 in the cortex and spinal cord.

\section{Widespread Neurodegeneration upon TDP-43 ${ }^{\text {A315T }}$ Expression in Mature Mice}

Constitutive expression of TDP-43 ${ }^{\mathrm{A} 315 \mathrm{~T}}$ from birth and throughout brain development resulted in early-onset neurodegeneration in iTDP- $43^{\mathrm{A} 315 \mathrm{~T}}$ mice. ${ }^{10}$ To assess mutant TDP-43 expression in matured brains, transgene expression was induced in iTDP- $43^{\mathrm{A3} 15 \mathrm{~T}}$ mice after weaning with littermate controls and aged till 15 months of age for histologic analysis. These diTDP-43 mice showed significant hippocampal degeneration with reduced area and increased 


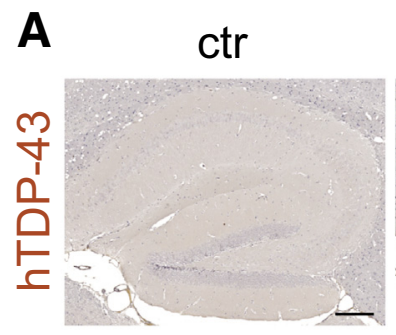

diTDP-43

B

ctr

diTDP-43
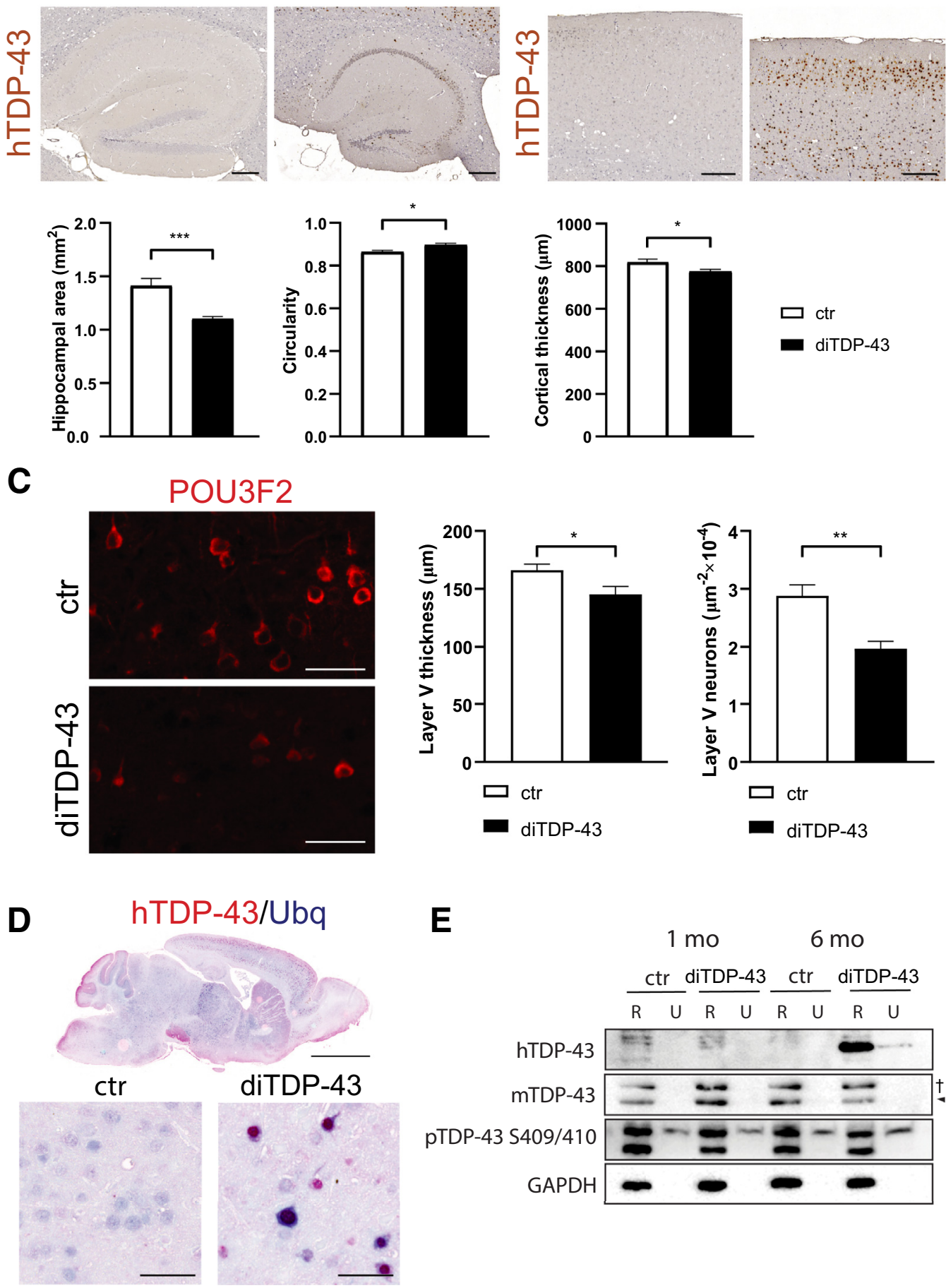

$\mathbf{E}$

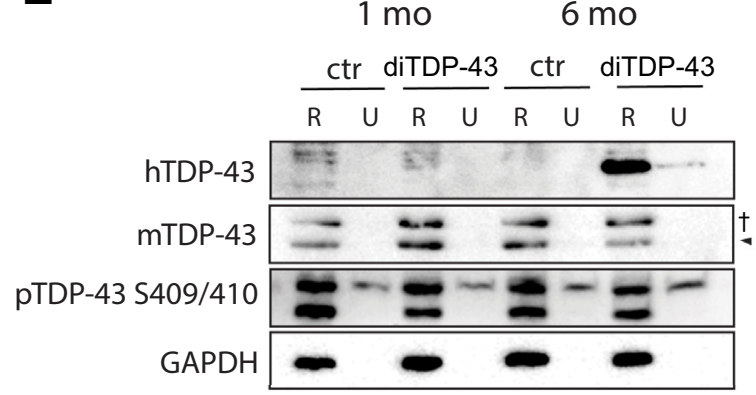

Figure 2 A: Human TAR DNA binding protein 43 (hTDP-43; brown) staining in transgenic diTDP-43 and control mice shows marked reduction in hippocampus size (top row) as quantified by cross-sectional area (bottom left panel). This reduction is more pronounced along the anteroposterior axis, resulting in increased circularity of hippocampal cross section (bottom right panel). B: hTDP-43 staining in the cortices of diTDP-43 and control mice reveals cortical reduction (top row), as quantified by cortical thickness (bottom panel). C: Immunofluorescence staining with layer II/III/V marker POU class 3 homeobox 2 (POU3F2) (red) reveals a reduction in layer V pyramidal neurons (left panels), resulting in a decrease in layer V thickness (middle panel) and neuronal density (right panel). D: Double chromogenic staining with hTDP-43 (red) and ubiquitin (blue) of diTDP-43 mice and a representative image of whole-brain scan as shown (top panel). Ubiquitination is observed in neurons expressing pathologic hTDP-43 (bottom panels). E: Extraction of proteins with radioimmunoprecipitation assay RIPA (R) and urea (U) buffers from 1- and 6-month-old diTDP-43 versus control (ctr) mice shows transgene expression at 6 months (hTDP-43) with a concomitant small decrease in murine TDP-43 (mTDP-43; arrowhead). The dagger indicates an unspecific band detected by the mTDP-43 antibody. Increased insolubility of hTDP-43 is observed with no changes in levels of phospho-TDP-43 serine 409/410. GAPDH is used as loading control. ${ }^{*} P<0.05,{ }^{*} P<0.01$, and ${ }^{* *} P<0.001$. Scale bars: $200 \mu \mathrm{m}$ (A and B); $50 \mu \mathrm{m}$ (C, and $\mathbf{D}$, bottom panels); $2 \mu \mathrm{m}$ (D, top panel). 

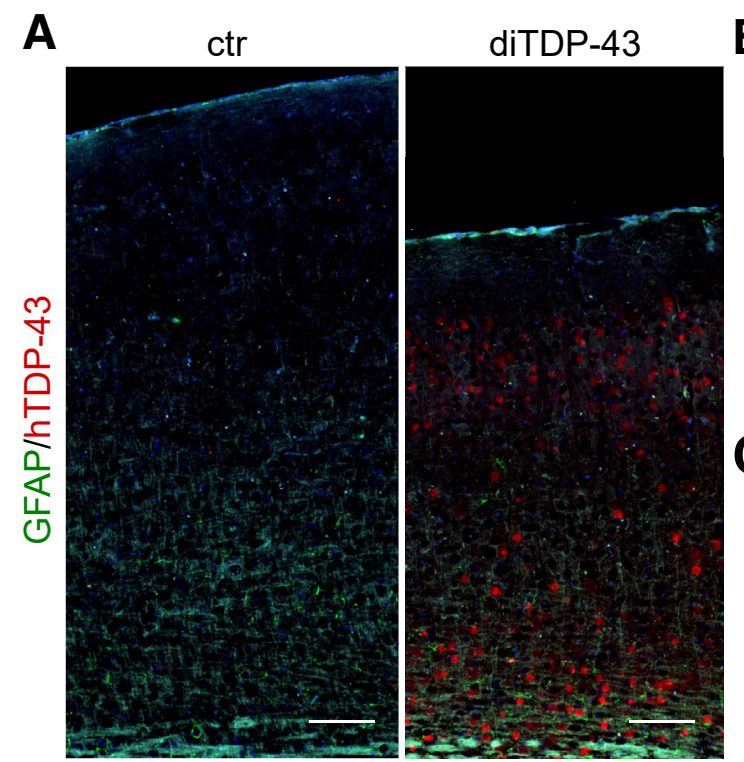

B

D

diTDP-43

C
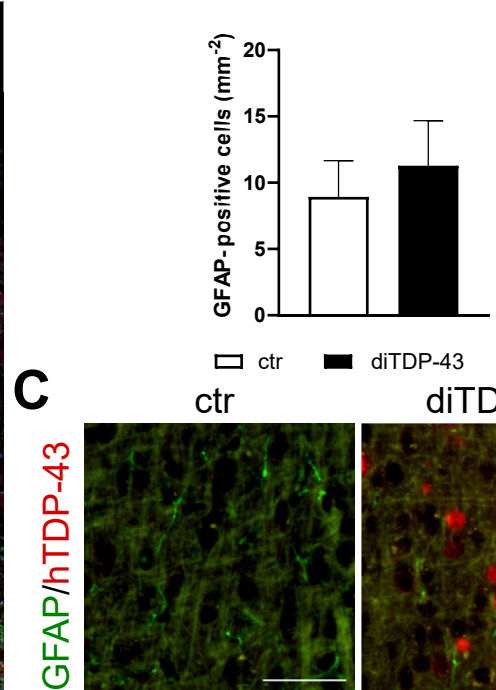

diTDP-43

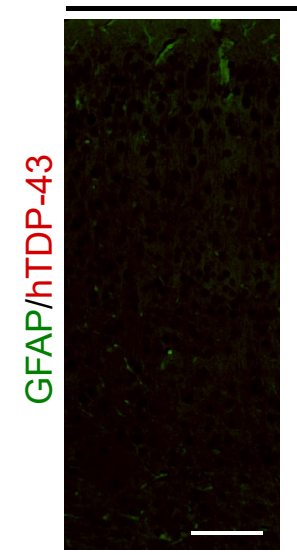

Age

1

\section{E}
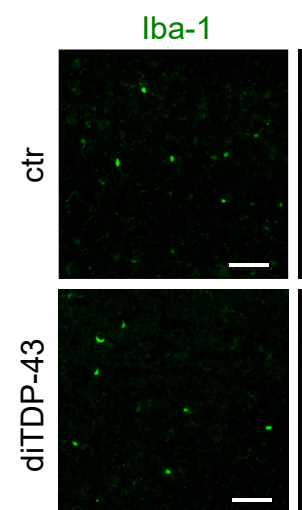

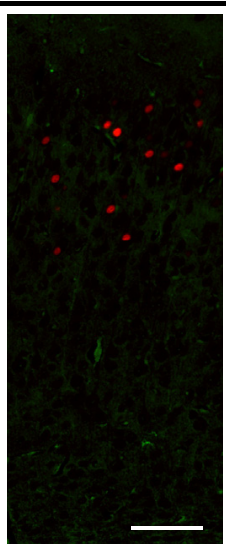

2

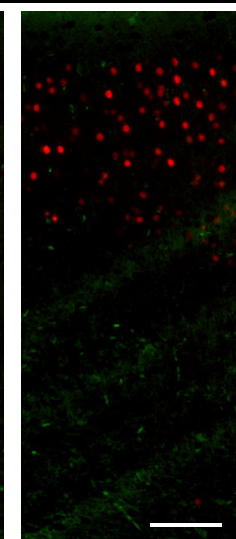

4

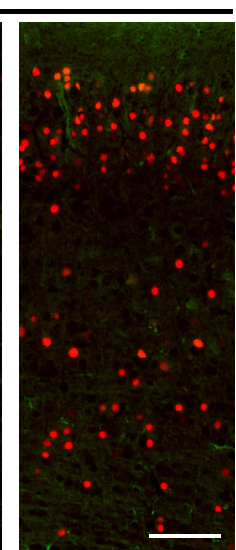

6

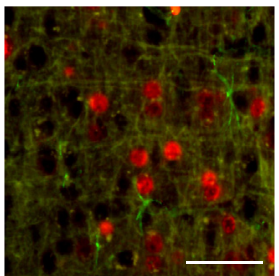

ctr

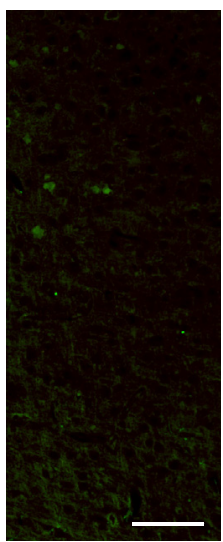

6
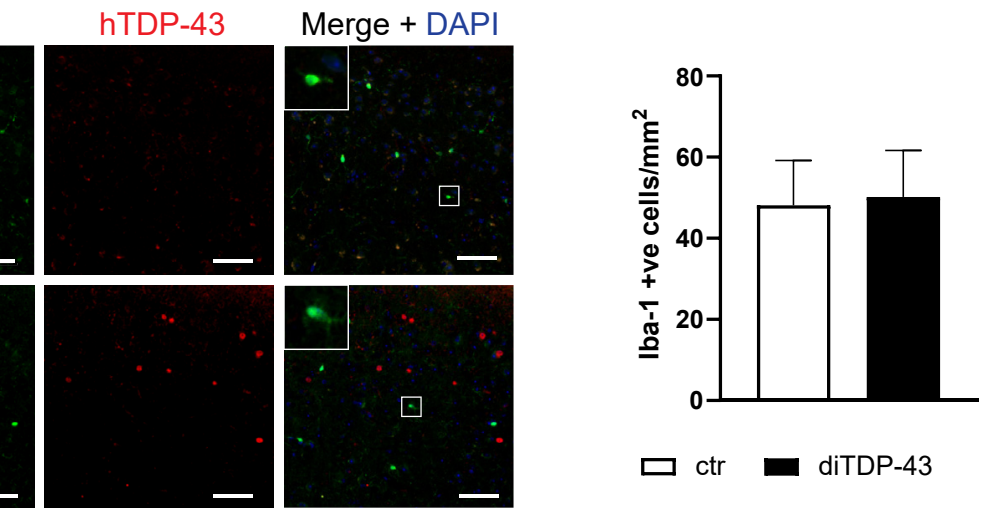

Figure 3 A: Immunofluorescence colabeling with antibodies against glial fibrillary acidic protein (GFAP) (green) and human TAR DNA binding protein (red) shows no significant differences between transgenic diTDP-43 and control (ctr) mice. B: Quantification in the cortex shows no significant differences in the number of GFAP-positive cells between diTDP-43 and control mice. C: No apparent differences are observed in the morphology of GFAP-positive cells in diTDP-43 and control mice. D: Representative images of diTDP-43 and control mice show absence of GFAP reactivity (green) from 1 to 6 months in diTDP-43 mice despite increase in transgene expression (hTDP-43; red). E: Double immunofluorescence staining of 12-month-old diTDP-43 (hTDP-43; red) and control brains show no difference in Iba-1 reactivity (green) and morphology (inset). Boxed areas are shown at higher magnification in the insets. Quantification of Iba-1-positive cells confirms no differences in numbers (right). Scale bars: $100 \mu \mathrm{m}$ (A and D); $50 \mu \mathrm{m}$ (C and E). Iba-1, ionized calcium binding adapter molecule. 
circularity (ratio of anterior-posterior length to dorsal-ventral length) as compared with littermate controls (Figure 2A). Similarly, cortical thickness was significantly reduced in 15-month-old diTDP-43 mice after delayed induction of transgene expression (Figure 2B). Accordingly, when brain sections were stained with the cortical neuronal layer marker POU3F2, ${ }^{12}$ a significant reduction of the numbers of layer $\mathrm{V}$ neurons together with a corresponding decrease in layer $\mathrm{V}$ thickness was found (Figure 2C). Immunohistochemical double labelling of brain sections showed costaining of neurons for human transgenic TDP-43 and cytoplasmic ubiquitin in diTDP-43 mice, whereas there was no corresponding staining in controls (Figure 2D). In assessing solubility of TDP-43 by extracting cortical brain tissue using increasingly stringent conditions, high levels of RIPA-soluble hTDP-43 were found to be associated with insoluble hTDP-43 detected in the urea fraction 6 months after transgene induction (Figure 2E). However, there were no overt changes in TDP-43 phosphorylation at serine 409/ 410 observed. Consistent with slow induction of hTDP-43 expression in diTDP-43 mice (Figure 1B), hTDP-43 levels were not detectable 1 month after transgene induction. The decrease of murine TDP-43 after 6 months of hTDP-43 was limited (Figure 2E), in line with the limited numbers of hTDP-43-expressing cells at this time (Figure 1B). Taken together, this study found significant neurodegeneration and TDP-43 neuropathology in aged TDP-43 ${ }^{\mathrm{A} 315 \mathrm{~T}}$ mice after delayed transgenic expression.

\section{Absence of Overt Astrogliosis upon TDP-43 ${ }^{\text {A315T }}$ Expression in Mature Mice}

Constitutive transgenic TDP-43 expression and neurodegeneration were accompanied by a pronounced and persisting astrogliosis in iTDP-43 ${ }^{\mathrm{A} 315 \mathrm{~T}}$ mice. ${ }^{10}$ To determine whether there was a similar astrogliosis in iTDP$43^{\mathrm{A} 315 \mathrm{~T}}$ mice after delayed transgene expression, brains were stained with the astrocyte marker GFAP. Surprisingly, similar astrocyte labelling in diTDP-43 mice compared with control brains were detected despite the pronounced neurodegeneration present in 15-month-old diTDP-43 mice (Figure 3A). Accordingly, quantification of GFAP-positive cells showed comparable numbers in diTDP-43 mice and control brains (Figure 3B), and the morphology of astrocytes was indistinguishable in all genotypes (Figure 3C). Staining of brains with GFAP and human TDP-43 antibodies from 1 to 6 months of age furthermore showed that astrogliosis was absent, whereas transgenic TDP- $43^{\mathrm{A} 315 \mathrm{~T}}$ expression increased over time (Figure 3D). No overt differences in microglial reactivity were found (Figure 3E), similar to what was previously observed in constitutive expression in iTDP-43 ${ }^{\mathrm{A} 315 \mathrm{~T}}$ mice. ${ }^{10}$ In short, delayed transgenic human TDP-43 expression in diTDP-43 mice was not associated with an overt astro- or microgliosis.
Motor Deficits and Muscle Atrophy after Delayed Transgene Expression in iTDP-43 ${ }^{\mathrm{A} 315 \mathrm{~T}}$ Mice

Constitutive transgenic TDP-43 expression in iTDP-43 ${ }^{\mathrm{A} 315 \mathrm{~T}}$ mice was associated with early-onset motor/gait deficits, muscle weakness, and muscle atrophy. ${ }^{10,12}$ To determine whether delayed transgene expression resulted in similar motor system dysfunction in iTDP-43 ${ }^{\mathrm{A} 315 \mathrm{~T}}$ mice, 9-monthold transgene-expressing and control mice were subjected to different motor test paradigms. During pole testing, diTDP43 mice required significantly more time to descend as compared with controls (Figure 4A). Similarly, wire testing revealed a significantly shortened time to fall in diTDP-43 mice as compared with controls (Figure 4B). Consistent with a reduced muscle performance, fore-limb grip strength was significantly reduced in diTDP-43 mice as compared with controls (Figure 4C). During rotarod testing, diTDP-43 mice presented only with moderately, but not significantly, reduced performance as compared with controls (Figure 4D). Digital gait analysis revealed significantly reduced stride time and length in diTDP-43 mice as compared with controls (Figure 4E). Staining of muscle cross sections with the basal membrane marker laminin, followed by segmentation for quantitative analysis demonstrated smaller muscle fibers in diTDP-43 mice as compared with controls (Figure 4F). Quantification confirmed significantly reduced muscle fiber areas in diTDP-43 mice as compared with controls and altered distribution of fiber diameters with loss of large diameter fibers and increased numbers of small diameter fibers (Figure 4G). Taken together, delayed transgene expression resulted in muscle atrophy and motor/gait deficits in aged diTDP-43 mice.

\section{Discussion}

In the present study, the analysis of iTDP-43 ${ }^{\mathrm{A} 315 \mathrm{~T}}$ mice was extended to expressing transgenic human TDP-43 only after brain development and maturation has completed. In these mice, neuronal TDP-43 pathology was associated with overt neurodegeneration, muscle atrophy, and motor/gait deficits. In contrast to iTDP-43 ${ }^{\mathrm{A} 315 \mathrm{~T}}$ mice with constitutive expression throughout brain maturation with pronounced and persisting astrogliosis, delayed initiation of transgene expression did not result in overt astrogliosis despite neurodegeneration and functional impairment.

When comparing the functional deficits and neurodegeneration upon delayed transgene expression in iTDP$43^{\mathrm{A} 315 \mathrm{~T}}$ mice (this study) with the motor impairments and neuron loss presented by iTDP- $43^{\mathrm{A} 315 \mathrm{~T}}$ mice with expression during development and brain maturation,,${ }^{10,12}$ no overt differences were observed. Accordingly, both constitutive and delayed transgene expression resulted in loss of layer $\mathrm{V}$ neurons in the cortex and pronounced hippocampal neuron loss, together with muscle atrophy and reduced performance across different motor paradigms. The lack of a significant 
A

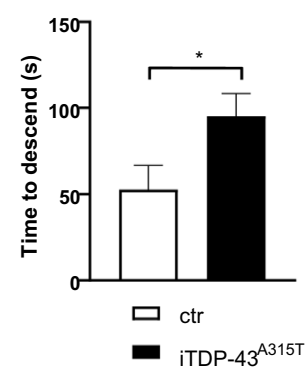

B

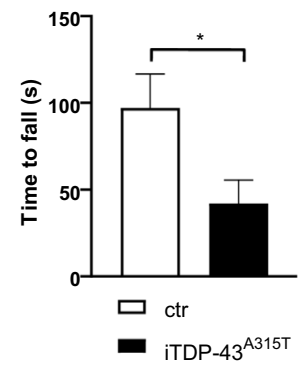

C

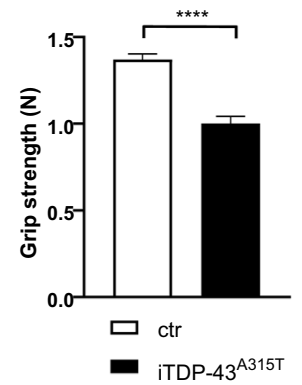

D

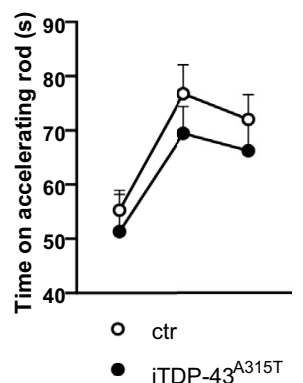

E
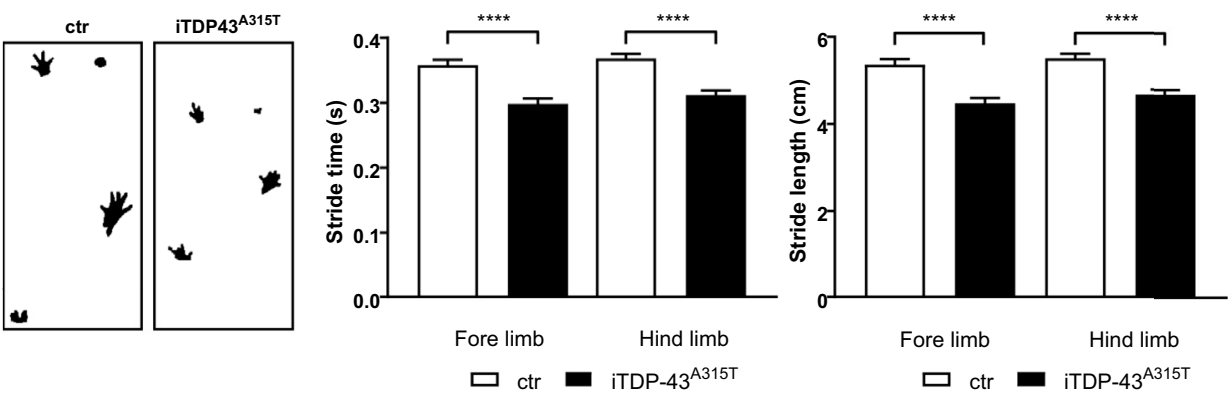

F

Laminin

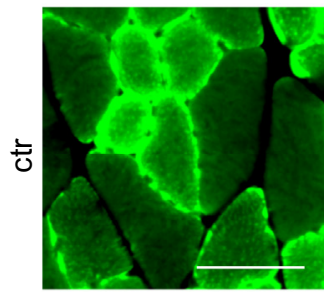

Segmentation

G
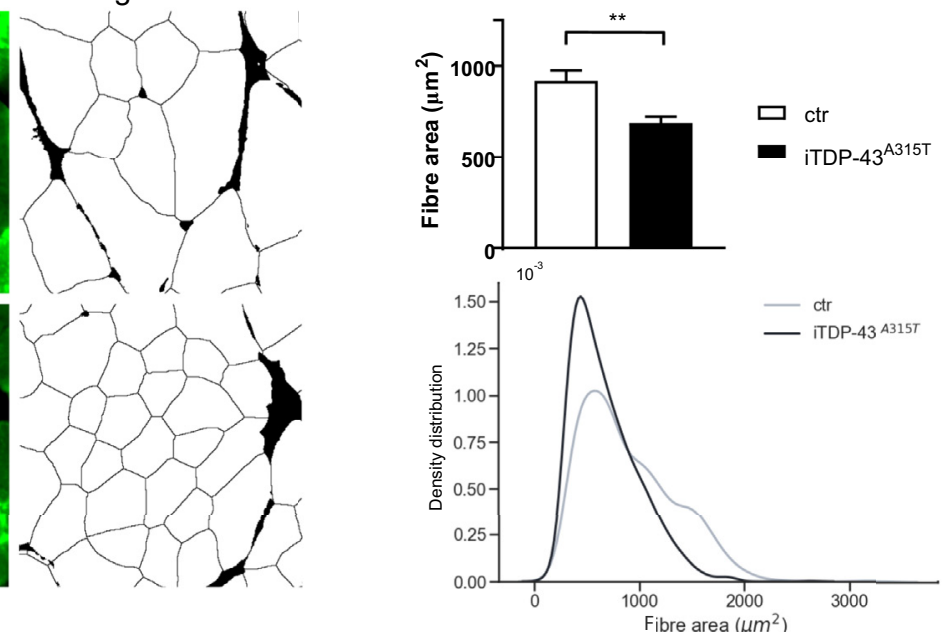

Figure 4 A: Transgenic diTDP-43 mice take significantly more time to descend on the vertical pole test as compared with control (ctr). B: diTDP-43 mice are faster to fall off on the inverted hanging wire test as compared with ctr. C: diTDP-43 have less forearm grip strength as compared with ctr. D: No significant differences are observed on the rotarod. E: Representative paw traces taken of diTDP-43 and control mice (left panel) show reduced stride time (middle panel) and stride length (right panel) in diTDP-43 mice, indicating gait abnormalities. F: Immunofluorescence staining with anti-laminin antibody (green) shows an increase in small muscle fibers in diTDP-43 mice compared with ctr. G: Segmentation and quantification show a significant reduction in crosssectional area of individual muscle fibers (top panel) with a reduction in large caliper muscle fibers (kernel density estimation, below). $n=13$ (A to $\mathbf{E}$, diTDP-43); $n=14$ (A to E, pooled ctr). ${ }^{*} P<0.05,{ }^{* *} P<0.01$, and ${ }^{* * *} P<0.0001$. Scale bar $=50 \mu \mathrm{m}$.

difference in rotarod performance between iTDP-43 ${ }^{\mathrm{A} 315 \mathrm{~T}}$ mice with delayed transgene expression versus controls is likely due to the poor overall performance of aged mice not previously exposed to the paradigm. Together, these data suggest that the severity of functional deficits and neurodegeneration in iTDP- $43^{\mathrm{A} 315 \mathrm{~T}}$ mice is not modulated by the central nervous system maturation state at the time of transgene induction, whereas the progression depends on its levels and/or timing. These findings differ from earlier inducible human nonmutant TDP-43 mice that showed pronounced neurodegeneration when constitutively expressed, whereas degeneration after transgene expression initiated at weaning was similarly rapid occurring, and did not present as pronounced over time as compared with the constitutive model. ${ }^{16}$ Similarly, transgenic TDP-43 expression was rapidly induced after doxycycline withdrawal at weaning in inducible mice expressing low levels of nonmutant TDP-43 or high levels of a deletion variant that 
localizes exclusively to the cytoplasm as well as using different inducer lines. ${ }^{17,18}$ Interestingly, neurodegeneration was similarly fast and extensive in these models, suggesting that toxic pathways do not correlate with expression levels. To this end, the delayed iTDP- $43^{\mathrm{A} 315 \mathrm{~T}}$ mice in the current study differs from the previous models by a comparatively slower increase in transgene expression after induction. ${ }^{16-18}$ This may be due to the use of alternative inducer lines of others: CamKII promoter ${ }^{16,17}$ or the NEFH promoter $^{18}$ as compared with the mThy 1.2 promoter-driven system used in this study. ${ }^{13}$ Furthermore, given that the motor impairments, and brain and muscle atrophy were comparable between the constitutive and delayed transgene-expressing iTDP- $43^{\mathrm{A} 315 \mathrm{~T}}$ mice, with absence of astrogliosis in the latter, the authors propose that the deficits observed are driven by neuronal pathways rather than by astrocytes.

Astrocytes become activated in response to virtually all pathologic conditions of the central nervous system, including in ALS and FTLD. ${ }^{19,20}$ Although the reactive response to the pathologic environment may be beneficial at onset of disease, the current understanding is that prolonged astrocytosis contributes to neuronal dysfunction and degeneration. ${ }^{21}$ In both human ALS and murine models of the disease, activated astrocytes were found before onset of motor systems, in the grey matter of the spinal cord and the cortex, as well as in the corticospinal tracts and the subcortical white matter. ${ }^{19}$ Although astrogliosis was not seen after delayed transgene expression in iTDP- $43^{\mathrm{A} 315 \mathrm{~T}}$ mice, other inducible TDP-43 transgenic mice showed late astrogliosis associated with a more rapid transgene increase after doxycycline withdrawal. ${ }^{16}$ This suggests that the pronounced astrogliosis in iTDP-43 ${ }^{\mathrm{A} 315 \mathrm{~T}}$ mice and other models with constitutive TDP-43 expression throughout brain maturation ${ }^{10,16}$ may be a secondary response to the rapid neurodegeneration observed during a vulnerable period and persists once initiated. Alternatively, astrogliosis in these mice could be a result of rapidly increased TDP-43 expression. Further studies are needed to decipher the signals between neurons, astrocytes, and microglia in TDP-43 pathology.

In summary, this study observed that functional deficits induced by a slow increase in expression of human mutant TDP-43 after brain maturation focused on neuronal dysfunction, in the absence of overt astrogliosis. This may suggest that the progressive neurodegeneration associated with TDP-43 pathology in FTLD and ALS is due to cellintrinsic disease pathways, and glial reactivity is a secondary response. To this end, further research is required to determine how these findings translate to the role of astrogliosis in human FTLD and ALS.

\section{References}

1. Burrell JR, Halliday GM, Kril JJ, Ittner LM, Gotz J, Kiernan MC, Hodges JR: The frontotemporal dementia-motor neuron disease continuum. Lancet 2016, 388:919-931

2. Neumann M, Sampathu DM, Kwong LK, Truax AC, Micsenyi MC, Chou TT, Bruce J, Schuck T, Grossman M, Clark CM, McCluskey LF,
Miller BL, Masliah E, Mackenzie IR, Feldman H, Feiden W, Kretzschmar HA, Trojanowski JQ, Lee VM: Ubiquitinated TDP-43 in frontotemporal lobar degeneration and amyotrophic lateral sclerosis. Science 2006, 314:130-133

3. Josephs KA, Whitwell JL, Weigand SD, Murray ME, Tosakulwong N, Liesinger AM, Petrucelli L, Senjem ML, Knopman DS, Boeve BF, Ivnik RJ, Smith GE, Jack CR Jr, Parisi JE, Petersen RC, Dickson DW: TDP-43 is a key player in the clinical features associated with Alzheimer's disease. Acta Neuropathol 2014, 127:811-824

4. Buratti E, Baralle FE: Multiple roles of TDP-43 in gene expression, splicing regulation, and human disease. Front Biosci 2008, 13: 867-878

5. Winton MJ, Igaz LM, Wong MM, Kwong LK, Trojanowski JQ, Lee VM: Disturbance of nuclear and cytoplasmic TAR DNA-binding protein (TDP-43) induces disease-like redistribution, sequestration, and aggregate formation. J Biol Chem 2008, 283:13302-13309

6. Zhang YJ, Xu YF, Cook C, Gendron TF, Roettges P, Link CD, Lin WL, Tong J, Castanedes-Casey M, Ash P, Gass J, Rangachari V, Buratti E, Baralle F, Golde TE, Dickson DW, Petrucelli L: Aberrant cleavage of TDP-43 enhances aggregation and cellular toxicity. Proc Natl Acad Sci U S A 2009, 106:7607-7612

7. Iguchi Y, Katsuno M, Niwa J, Takagi S, Ishigaki S, Ikenaka K, Kawai K, Watanabe H, Yamanaka K, Takahashi R, Misawa H, Sasaki S, Tanaka F, Sobue G: Loss of TDP-43 causes agedependent progressive motor neuron degeneration. Brain 2013, 136:1371-1382

8. Tan RH, Ke YD, Ittner LM, Halliday GM: ALS/FTLD: experimental models and reality. Acta Neuropathol 2017, 133:177-196

9. Kabashi E, Valdmanis PN, Dion P, Spiegelman D, McConkey BJ, Vande Velde C, Bouchard JP, Lacomblez L, Pochigaeva K, Salachas F, Pradat PF, Camu W, Meininger V, Dupre N, Rouleau GA: TARDBP mutations in individuals with sporadic and familial amyotrophic lateral sclerosis. Nat Genet 2008, 40:572-574

10. Ke YD, van Hummel A, Stevens CH, Gladbach A, Ippati S, Bi M, Lee WS, Kruger S, van der Hoven J, Volkerling A, Bongers A, Halliday G, Haass NK, Kiernan M, Delerue F, Ittner LM: Shortterm suppression of A315T mutant human TDP-43 expression improves functional deficits in a novel inducible transgenic mouse model of FTLD-TDP and ALS. Acta Neuropathol 2015, 130: 661-678

11. Ittner LM, Halliday GM, Kril JJ, Gotz J, Hodges JR, Kiernan MC: FTD and ALS-translating mouse studies into clinical trials. Nat Rev Neurol 2015, 11:360-366

12. van Hummel A, Chan G, van der Hoven J, Morsch M, Ippati S, Suh L, Bi M, Asih PR, Lee WS, Butler TA, Przybyla M, Halliday GM, Piguet O, Kiernan MC, Chung RS, Ittner LM, Ke YD: Selective spatiotemporal vulnerability of central nervous system neurons to pathologic TAR DNA-binding protein 43 in aged transgenic mice. Am J Pathol 2018, 188:1447-1456

13. Delerue F, White M, Ittner LM: Inducible, tightly regulated and nonleaky neuronal gene expression in mice. Transgenic Res 2014, 23: 225-233

14. Ke YD, Delerue F, Gladbach A, Götz J, Ittner LM: Experimental diabetes mellitus exacerbates tau pathology in a transgenic mouse model of Alzheimer's disease. PLoS One 2009, 4:e7917

15. Schindelin J, Arganda-Carreras I, Frise E, Kaynig V, Longair M, Pietzsch T, Preibisch S, Rueden C, Saalfeld S, Schmid B, Tinevez JY, White DJ, Hartenstein V, Eliceiri K, Tomancak P, Cardona A: Fiji: an open-source platform for biological-image analysis. Nat Methods 2012, 9:676-682

16. Cannon A, Yang B, Knight J, Farnham IM, Zhang Y, Wuertzer CA, D'Alton S, Lin WL, Castanedes-Casey M, Rousseau L, Scott B, Jurasic M, Howard J, Yu X, Bailey R, Sarkisian MR, Dickson DW, Petrucelli L, Lewis J: Neuronal sensitivity to TDP-43 overexpression is dependent on timing of induction. Acta Neuropathol 2012, 123: 807-823 
17. Igaz LM, Kwong LK, Lee EB, Chen-Plotkin A, Swanson E, Unger T, Malunda J, Xu Y, Winton MJ, Trojanowski JQ, Lee VM: Dysregulation of the ALS-associated gene TDP-43 leads to neuronal death and degeneration in mice. J Clin Invest 2011, 121:726-738

18. Walker AK, Spiller KJ, Ge G, Zheng A, Xu Y, Zhou M, Tripathy K, Kwong LK, Trojanowski JQ, Lee VM: Functional recovery in new mouse models of ALS/FTLD after clearance of pathological cytoplasmic TDP-43. Acta Neuropathol 2015, 130: $643-660$
19. Ben Haim L, Carrillo-de Sauvage MA, Ceyzeriat K, Escartin C: Elusive roles for reactive astrocytes in neurodegenerative diseases. Front Cell Neurosci 2015, 9:278

20. Phatnani H, Maniatis T: Astrocytes in neurodegenerative disease. Cold Spring Harb Perspect Biol 2015, 7:a020628

21. Radford RA, Morsch M, Rayner SL, Cole NJ, Pountney DL, Chung RS: The established and emerging roles of astrocytes and microglia in amyotrophic lateral sclerosis and frontotemporal dementia. Front Cell Neurosci 2015, 9:414 